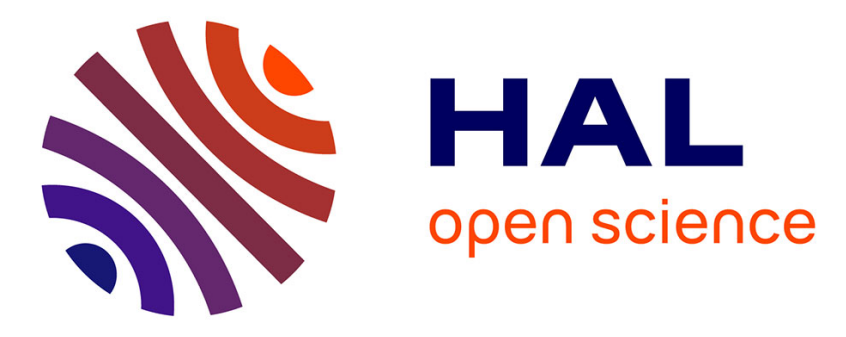

\title{
Optimal orientation estimators for detection of cylindrical objects
}

Christianne Mulat, Marc Donias, Pierre Baylou, Gérard Vignoles, Christian

Germain

\section{- To cite this version:}

Christianne Mulat, Marc Donias, Pierre Baylou, Gérard Vignoles, Christian Germain. Optimal orientation estimators for detection of cylindrical objects. Signal, Image and Video Processing, 2008, 2 (1), pp.51-58. 10.1007/s11760-007-0035-2 . hal-00188509

\section{HAL Id: hal-00188509 \\ https://hal.science/hal-00188509}

Submitted on 17 Nov 2007

HAL is a multi-disciplinary open access archive for the deposit and dissemination of scientific research documents, whether they are published or not. The documents may come from teaching and research institutions in France or abroad, or from public or private research centers.
L'archive ouverte pluridisciplinaire HAL, est destinée au dépôt et à la diffusion de documents scientifiques de niveau recherche, publiés ou non, émanant des établissements d'enseignement et de recherche français ou étrangers, des laboratoires publics ou privés. 
Title:

\section{Optimal orientation estimators for detection of cylindrical objects}

Running head :

Gradient for detecting cylinder axis

Keywords: image processing, cylinder, orientation, gradient masks, 3D.

Manuscript size: 11 pages, 1 table, 11 figures

Authors:

Christianne Mulat

LCTS, UMR 5801 CNRS-UB1-Safran-CEA,

3 allée La Boëtie, Domaine Universitaire, 33600 Pessac, France.

Tel +33 56844734 Fax. +3356841225

Email.mulat@lcts.u-bordeaux1.fr.

Marc Donias

IMS, Dept. LAPS, Equipe Signal et Image, UMR 5218 CNRS ENSEIRB,

Bâtiment A4, 351 cours de la libération, 33405 Talence, France.

Tel. +33 540003623 Fax. +33 540006644

Email.marc.donias@laps.ims-bordeaux.fr

Pierre Baylou,

IMS, Dept. LAPS, Equipe Signal et Image, UMR 5218 CNRS - ENSEIRB,

Bâtiment A4, 351 cours de la libération, 33405 Talence, France.

Tel. +33 540006142 Fax. +33 540006644

Gérard Vignoles

LCTS, UMR 5801 CNRS-UB1-Safran-CEA,

3 allée La Boëtie, Domaine Universitaire, 33600 Pessac, France.

Tel +33 56844734 Fax. +3356841225

Email.vinhola@lcts.u-bordeaux1.fr

Christian GERMAIN

IMS, Dept. LAPS, Equipe Signal et Image, UMR 5218 CNRS -ENITAB,

Bâtiment A4, 351 cours de la libération, 33405 Talence, France.

Tel. +33 540006142 Fax. +33 440006644

Email.christian.germain@laps.ims-bordeaux.fr 
Abstract: This paper introduces low level operators in the context of detecting cylindrical axis in 3-D images. Knowing the axis of a cylinder is particularly useful since cylinder location, length and curvature derive from this knowledge. This paper introduces a new gradient-based optimal operator dedicated to accurate estimation of the direction toward the axis. The operator relies on Finite Impulse Response filters. The approach is presented first in a 2-D context, thus providing optimal gradient masks for locating the center of circular objects. Then, a 3-D extension is provided, allowing the exact estimation of the orientation toward the axis of cylindrical objects when this axis coincides with one of the mask reference axes. Applied to more general cylinders and to noisy data, the operator still provides accurate estimation and outperforms classical gradient operators.

Mailing address for galley proofs.

Christian GERMAIN

IMS, Dept. LAPS, Equipe Signal et Image,

Bâtiment A4, 351 cours de la libération, 33405 Talence, France.

Tel. +33 540006142 Fax. +33 540006644

Email.christian.germain@laps.ims-bordeaux.fr 


\section{Introduction}

3-D Image acquisition techniques allow characterizing effectively the internal structure of material [1] or finely depicting organ shape in medical imaging [2]. Among the structures which are found within such 3-D data, tubular and cylindrical objects are especially of interest, since they appear in fibrous composite material (carbon or glass fibers) [3] as well as in angiography (blood vessels) [4]. Characterizing the shape of such objects mainly consists in locally determining both axis and section. Knowing the axis is particularly useful because cylinder location, length and curvature derive from it.

The axis of a cylindrical object can be detected in two distinct ways. On one hand, the objects of interest can be detected or segmented from their background [4][6][7]; the axis of each component is then estimated. This approach is suitable when voxels within the cylindrical objects are homogeneous and distinguishable from the background. Image segmentation algorithms constitute the first step of such an approach [8][9]. Axis detection then derives from statistics or mathematical morphology. Algorithms based on the Hough transform of the voxel cloud resulting from the segmentation step have also been successfully used for this purpose [10][11][12].

On the other hand, the axis of each cylindrical object can be detected directly from the 3-D data, without preliminary segmentation of the objects. In this case, it should be noted that once the axis is detected, a cylinder detection algorithm can retrieve the whole cylinder using the axis as an initialization step [13][14][15][16]. Since this kind of approach does not need any pre-segmentation, this is an appropriate choice if the cylindrical objects are not homogeneous, or if the voxel grey levels are similar to those of the background (see Figure a). Detecting the axis of the cylinder directly from image intensity can be achieved by estimating, from each voxel, the direction in which the axis will be found. Hereafter, this direction will be referred to as the orientation toward the cylinder axis [17]. Estimating the orientation toward the axis relies on computing the derivatives of image intensity. For discrete images, the derivatives cannot be directly computed but are usually estimated using the convolution between dedicated masks and the grey level data. Classical gradient masks can be found in the literature. Among them, Prewitt and Sobel masks are frequently used with 2-D 
images [18] and can be easily extended to 3-D. However, they are not well adapted to orientation estimation in the case of cylindrical objects as they provide biased estimation. Other gradient estimators have been optimized regarding some specific criteria. For example, Canny [19] proposes well-known gradient masks optimized for the detection of a step profile in white Gaussian noise. Le Pouliquen [20] also proposes unbiased masks for estimating the orientation of 2-D directional textures.

This paper proposes new gradient masks for the estimation of gradient direction with a view to obtaining the most accurate axis location of cylinders in 3-D images. Theoretical developments were carried out starting from cylindrical objects for which the axis coincided with one of the mask reference axes. In the following sections, such cylindrical objects will be referred to as vertical cylinders. First, our approach is introduced for 2-D images, in the case of circular objects with monomial profile functions such as $f(r)=r^{k}$. Relations between mask coefficients are established and 2-D masks are proposed. In section 3, the previous 2-D approach is extended to 3-D for vertical cylinders, and 3-D masks are proposed. The 3-D masks provided are applied to both synthetic and real data in the fourth section. Experiments are extended to any cylindrical object and the noise sensitivity of the proposed masks is also addressed.

\section{2-D unbiased masks for radial objects}

\subsection{Profile function}

Let us consider a 2-D case, i.e. the orthogonal cross section of a 3-D cylindrical object. The corresponding disk is described by its profile function $f(r)=r^{N}$ with $r=\sqrt{x^{2}+y^{2}}$. Figure b illustrates the case $f(r)=r^{8}$.

For each pixel, the direction toward the axis of the cylinder becomes the direction toward the center of the disk and is given by the gradient orientation $\theta$ :

$$
\theta=\arctan \left(\frac{G_{y}}{G_{x}}\right)=\arctan \left(\frac{y}{x}\right)
$$

with $G_{x}=\frac{\partial f}{\partial x}=\frac{x}{\sqrt{x^{2}+y^{2}}} \frac{d f}{d r}$ and $G_{y}=\frac{\partial f}{\partial y}=\frac{y}{\sqrt{x^{2}+y^{2}}} \frac{d f}{d r}$. 
Most functions $f(r)$ which have continuous derivatives up to order $N$ can be approximated by a polynomial of order $N$ using its Taylor expansion. Moreover, if the masks induce no bias for any monomial $f_{k}(r)=r^{k}(k \leq N)$, they induce no bias for the polynomial function $f(r)=\sum_{k=0}^{N} a_{k} r^{k}$.

Therefore, in this paper, the choice was made to design optimal masks for monomial functions $f_{N}(r)=r^{N}$, with order $N$ as high as possible. If $N$ is even, finding masks to obtain unbiased orientation estimation is an attainable goal. The profile functions considered are, for $N$ even:

$$
f(r)=r^{N}=r^{2 n}=\left(x^{2}+y^{2}\right)^{n}
$$

However, if $N$ is odd, the polynomial expansion of $\left(\sqrt{x^{2}+y^{2}}\right)^{N}$ is infinite, making it impossible to find masks which do not involve any bias with the profile function $f(r)$. In this case, optimal masks that minimize the orientation error will be sought.

\subsection{Convolution mask coefficients}

Let the $\vec{v}=\left(f_{x}, f_{y}\right)$ be the "orientation vector", i.e. any vector whose argument $\theta$ equals the orientation toward the center and such as $\|\vec{v}\| \neq 0$. Estimating the orientation $\theta$ then consists in finding two convolution masks $m_{x}$ and $m_{y}$, such that $f_{x}=f \otimes m_{x}$ and $f_{y}=f \otimes m_{y}$, and:

$$
\theta=\arctan \left(\frac{f_{y}}{f_{x}}\right) \equiv \arctan \left(\frac{y}{x}\right)
$$

Let $\left\{b_{i j} /(i, j) \in S^{+}\right\}$be the coefficients of the discrete mask, with $S^{+}$ corresponding to the right part ( $i>0$ ) of the graph (see Figure c for $m_{x}$ ) and $S^{-}$the left part $(i<0)$. Symmetries are imposed on the mask coefficients in order to carry out gradient estimation:

$$
\begin{array}{ll}
\checkmark & b_{-i j}=-b_{i j} \\
\checkmark & b_{i(-j)}=b_{i j} \\
\checkmark & b_{0 j}=0 \\
\checkmark & \forall(i, j) \notin S^{+} \cup S^{-}, b_{i j}=0
\end{array}
$$


When relation (3) is verified, the masks provide the exact orientation toward the center. Theses masks are referred to as unbiased masks. The following properties are proposed to assess whether a mask is biased or not. If one of these properties is satisfied, the corresponding masks give unbiased orientation estimation.

$$
\begin{gathered}
f_{x}=G_{x} \text { and } f_{y}=G_{y} \text { (4) } \\
f_{x}=\alpha \times x \text { and } f_{y}=\alpha \times y, \alpha \neq 0 \\
f_{x}=x \times \psi(x, y), f_{y}=y \times \psi(y, x) \text { and } \forall(x, y), \psi(x, y)=\psi(y, x)
\end{gathered}
$$

Conditions (4) and (5) are sufficient but too restrictive to be useful, while (6) is necessary and sufficient. Furthermore, it makes finding relations between the coefficients possible, in order to obtain unbiased orientation estimation.

For each point $(x, y)$ of the image, the discrete convolution can be written as:

$$
f_{x}=f \otimes m_{x}=\sum_{(i, j) \in S^{+}} b_{i j}[f(x+i, y+j)-f(x-i, y+j)]=\sum_{(i, j) \in S^{+}} b_{i j} \cdot \Delta_{i j},
$$

with $\Delta_{i j}=\left((x+i)^{2}+(y+j)^{2}\right)^{n}-\left((x-i)^{2}+(y+j)^{2}\right)^{n}$.

Using Newton's binomial theorem, the complete expansion of the components of the orientation vector $\vec{v}$ gives $f_{x}=2 x \sum_{r=0}^{n-1} \sum_{s=0}^{n-r-1} \alpha_{r s} x^{2 s} y^{2 r}, f_{y}=2 y \sum_{r=0}^{n-1} \sum_{s=0}^{n-r-1} \alpha_{r s} x^{2 r} y^{2 s}$, with:

$$
\begin{aligned}
& \alpha_{r s}=\left(\begin{array}{l}
n \\
r
\end{array}\right)\left(\begin{array}{l}
2(n-r) \\
2 s+1
\end{array}\right) \sum_{i \in N^{*}} b_{i 0} i^{2(n-r-s)-1} \\
& +2 \sum_{k=s+1}^{n-r}\left[\left(\begin{array}{l}
n \\
k
\end{array}\right)\left(\begin{array}{l}
2(n-k) \\
2 r
\end{array}\right)\left(\begin{array}{l}
2 k \\
2 s+1
\end{array}\right) \sum_{i \in N^{*}} \sum_{j \in N^{*}} b_{i j} j^{2(n-k-r)} i^{2(k-s)-1}\right]
\end{aligned}
$$

where $\left(\begin{array}{l}n \\ k\end{array}\right)=\frac{n !}{k ! \cdot(n-k) !}$ are the binomial coefficients.

To obtain $\frac{y}{x}=\frac{f_{y}}{f_{x}}$, the following existence condition (9) as well as the non-bias condition (10) have to be satisfied.

$$
\begin{gathered}
\exists r \in[0, n-1], \exists s \in[0, n-r-1], \alpha_{r s} \neq 0 \\
\forall r, s \quad \alpha_{r s}=\alpha_{s r}
\end{gathered}
$$

Combining condition (10) with equation (8) leads to establishing the relations between the mask coefficients. 


\subsection{Constraints for unbiased orientation estimation}

The constraints for unbiased orientation estimation are given below for $n=4$ and $n=5$. Let us note that considering higher values of $n$ is pointless, since a polynomial profile function of order $N=2 n=10$ leads to quasi binary images. As a result of the expansion, the following relations are obtained.

$n=4$

$$
\begin{aligned}
& \sum_{i \geq 1} i^{3}\left(b_{i 0}+2 \sum_{j \geq 1} b_{i j}\right)-6 \sum_{i \geq 1} \sum_{j \geq 1}\left(b_{i j} j^{2} i\right)=0 \\
& \sum_{i \geq 1} i^{5}\left(b_{i 0}+2 \sum_{j \geq 1} b_{i j}\right)-6 \sum_{i \geq 1} \sum_{j \geq 1}\left(b_{i j} j^{4} i\right)-4 \sum_{i \geq 1} \sum_{j \geq 1}\left(b_{i j} j^{2} i^{3}\right)=0
\end{aligned}
$$

$n=5$

$$
\begin{aligned}
& \sum_{i \geq 1} i^{3}\left(b_{i 0}+2 \sum_{j \geq 1} b_{i j}\right)-6 \sum_{i \geq 1} \sum_{j \geq 1}\left(b_{i j} j^{2} i\right)=0 \\
& 3 \sum_{i \geq 1} i^{5}\left(b_{i 0}+2 \sum_{j \geq 1} b_{i j}\right)-20 \sum_{i \geq 1} \sum_{j \geq 1}\left(b_{i j} j^{4} i\right)-10 \sum_{i \geq 1} \sum_{j \geq 1}\left(b_{i j} j^{2} i^{3}\right)=0 \\
& \sum_{i \geq 1} i^{7}\left(b_{i 0}+2 \sum_{j \geq 1} b_{i j}\right)-6 \sum_{i \geq 1} \sum_{j \geq 1}\left(b_{i j} j^{6} i\right)-10 \sum_{i \geq 1} \sum_{j \geq 1}\left(b_{i j} j^{4} i^{3}\right)-2 \sum_{i \geq 1} \sum_{j \geq 1}\left(b_{i j} j^{2} i^{5}\right)=0
\end{aligned}
$$

It can be easily proven that if the mask verifies relations (11) and (12), it is

unbiased for all profile functions defined by $f(r)=\left(\sqrt{x^{2}+y^{2}}\right)^{2 n} \forall n \leq 5$.

\subsection{Example of unbiased masks}

Given the constraints proposed in the preceding paragraph, unbiased masks can be established. The masks are centered on $O=(0,0)$ and are sketched at Figure d. Masks are termed GxDN where $x$ is the dimension of the data and $\mathrm{N}$ is the degree of the profile function.

The masks G2D10 satisfy (6), (11) and (12), so they induce no bias for the profile function for $N=2 n \leq 10$. These masks give estimates of orientation without bias for a circular profile.

\section{3-D unbiased masks for cylindrical objects}

Let the coordinate system be $(\vec{i}, \vec{j}, \vec{k})$. In order to facilitate the extension to the 3-D case, a vertical cylinder (with axis $\vec{k}$ ) is considered. The profile function becomes $f(x, y, z)=f(x, y)$. 


\subsection{Relations between 2-D and 3-D}

In the 3-D case, the positive and negative domains of a given mask will be noted $V^{+}$and $V^{-}$respectively. The link between $S^{+}$(2-D) and $V^{+}$(3-D) is given by $S^{+}=V^{+} \cap(k=0)$.

The coefficients of the mask are $\left\{a_{i j k} /(i, j, k) \in V^{+}\right\}$with the symmetry conditions imposed by gradient estimation as in the 2-D case.

The relation between mask coefficients in 2-D and in 3-D is:

$$
\forall(i, j) \in S^{+}, b_{i j}=\sum_{k \in Z} a_{i j k}
$$

This relation ensures that the unbiased 2-D masks can be extended to 3-D.

\subsection{Example of 3-D masks}

As an example, the extension in 3-D of the 2-D mask G2D10 (Figure d) is considered. This 2-D mask can be seen as a projective view of a 3-D mask, where the coefficients are the sum of the weights along the axis $k$ (relation (13)). Figure e illustrates the proposed 3-D mask, G3D10. Here, the coordinates of $O_{-2}$, $O_{-1}, O_{1}$ and $O_{2}$ are respectively $(-2,0,0),(-1,0,0),(1,0,0)$ and $(2,0,0)$. Note: For a complete representation of $m_{x}$, the same sections exist respectively in $i=-1$ and $i=-2$ as in $i=1$ and $i=2$, with negative coefficients.

\section{Results}

Various masks are tested in 3-D. In each case, the orientation bias (angle error) is evaluated. The results are compared to those of usual gradient masks.

\subsection{Error definition}

In 3-D, the estimation error $\Delta_{\theta}$ (Figure f) is given by the angle between the estimated orientation unit vector $\vec{v}_{n}=\vec{v} \cdot\|\vec{v}\|^{-1}$ and the unit vector normal to the cylinder axis $\vec{n}=\overrightarrow{M H} \cdot r^{-1}$, where $H$ is the orthogonal projection of the current point $M$ on the cylinder axis and $r=\|\overrightarrow{M H}\|$ :

$$
\left|\sin \left(\Delta_{\theta}\right)\right|=\left\|\vec{n} \wedge \vec{v}_{n}\right\|
$$




\subsection{Experimental conditions}

The gradients are estimated in 3-D synthetic blocks. The size of these blocks is $100 \times 100 \times 100$. The profile functions for defining the cylinders are:

$$
f(r)=r^{N} \text { and } r=\left(\sqrt{\left(x_{0}+\lambda y_{x}-x\right)^{2}+\left(y_{0}+\lambda u_{y}-y\right)^{2}\left(z_{0}+\lambda u_{z}-z\right)^{2}}\right)^{N}
$$

with $M_{0}=\left(x_{0}, y_{0}, z_{0}\right)$ and $\vec{u}=\left(u_{x}, u_{y}, u_{z}\right)=(\cos (\varphi) \cos (\theta), \cos (\varphi) \sin (\theta), \sin (\varphi))$. $\theta$ is the azimuth angle and $\varphi$ is the elevation angle. The axis equation (Figure $\mathrm{f}$ ) is defined by: $\left\{\overrightarrow{O M_{0}}+\lambda \vec{u}, \lambda \in \mathbb{R}\right\}$.

Hereafter, the experimental parameters for the profile functions are $M_{0}=(50,50,0)$, with $N=9$ or $N=10$ and various axis orientations are considered.

\section{Masks used for comparison}

In each case, the angle error obtained with G3D10 masks is compared to the error obtained with Prewitt (Figure g2), Sobel (Figure g3) and cross masks (Figure g4) under the same conditions. Figure g1 shows the 3-D shape of theses masks. These gradients estimators have been chosen since they are the most frequently used local gradients estimators. Others types of gradient estimators can be found [19][20] and have been designed in 2-D to be optimal for other specific criteria.

\subsection{Results for profile function of even order}

\section{Vertical cylinder axis}

The estimation error, as defined in section 4.1, is evaluated for each voxel of the block. Then, the maximal estimation error of the block is retained.

As expected, the maximal error for the G3D10 mask (Figure e) is negligible, $\Delta_{\theta \max }=10^{-6} \mathrm{deg} .$. Non-zero bias values are explained by the round-off and truncation errors involved during computation. In comparison, if the cross gradient is applied, the maximal error obtained is approximately $\Delta_{\theta \max }=8.9 \mathrm{deg}$. Applying Prewitt masks, the maximal error obtained is approximately $\Delta_{\theta \max }=6.7 \mathrm{deg}$. and for Sobel masks $\Delta_{\theta \max }=5.9 \mathrm{deg} .$. 


\section{Oblique cylinder axis}

Considering the case of oblique cylinders, the masks have been tested for various elevation angles $\varphi \in[0,180]$ with $\theta=90 \mathrm{deg}$. . The estimation error depends on the relative location with respect to the cylinder axis. Therefore, to compare mask sensitivity to axis elevation $\varphi$, the maximal error for all locations is computed and shown in Figure h. Table a presents the maximal, minimal and mean values for all locations and for all $\varphi$ values.

The first observation is that G3D10 is slightly biased when the axis does not correspond to a coordinate axis. An attempt was made to find mask coefficients corresponding to the minimal error, in the case of one specific axis orientation. Unfortunately, this was not possible since optimal coefficients are locationdependant, and, more specifically, depend on the distance from the axis. However, compared to cross, Sobel, and Prewitt masks, G3D10 mask gives much more accurate results (Table a).

\section{Noise robustness}

Gaussian white noises with various Signal to Noise Ratios (SNR) are added to the 3-D block with worse case axis elevation ( $\varphi=63 \mathrm{deg}$.). The SNR is defined by:

$$
S N R=20 \log \left(\frac{\operatorname{grad}_{r=3}}{\sigma_{b}}\right)
$$

where $\sigma_{b}$ is the standard deviation of the noise and $\operatorname{grad}_{r=3}$ is the gradient magnitude computed using only voxels for which the distance to the axis is $r=3 \pm 0.5$ pixel.

For each SNR considered, the maximal value is identified from the orientation errors of the selected voxels. In each case, the mean orientation error is then computed for 50 noise realizations.

As seen in Figure i, the mean orientation error obtained with the G3D10 mask is less than that obtained with the other masks, whatever the SNR ratio. G3D10 masks being slightly larger than Sobel, Prewitt and cross masks, this partly explains the better noise robustness of the proposed approach. 


\subsection{Results for profile function of odd order}

As seen in section 2.1, for odd order of the exponent $N$, the profile function has an infinite polynomial expansion. Therefore, it is not possible to fulfill the no-bias conditions. However, the G3D10 masks are tested in the case of profile functions of odd order $N=7$ and $N=9$. In each case and for one particular orientation, finding optimal mask coefficient values is attempted by estimating the error versus mask coefficient values. The coefficients of the G3D10 masks yield the minimum estimation error, which is less than $10^{-4} \mathrm{deg}$. This is a promising sign meaning that the proposed masks are quasi-optimal in the case of odd order profile functions.

Figure j presents the results obtained for $N=9$, using the same protocol as in section 4.3. This figure shows that, even in the case of profile function of odd order, the G3D10 masks gives better results than the other tested masks.

\subsection{Result for a fibrous composite material}

The G3D10 masks are now being tested on fibrous composite materials. 3-D blocks have been acquired at the ESRF (European Synchrotron Radiation Facility), ID19 beam line, using Synchrotron X-Ray Micro Tomography. Figure k shows the resulting vectors projected on the section of such a 3-D block. The arguments of these vectors gives the orientation toward the axes of the corresponding cylinders (modulo $\pi$ ). These estimations appear to be accurate within the cylinders. Outside the cylinders, the orientations are not significant, which is depicted by low magnitude gradients (magnitudes are not shown in Figure k).

\section{Conclusion}

In this paper, new convolution masks have been introduced to estimate the orientation toward the axis for cylindrical objects in 3D digital images. The approach presented relies on an optimal estimation of the derivatives of the profile function. These derivatives, which represent the grey level evolution inside the cylinder, are obtained convoluting gradient masks with the profile function. In establishing this model, the case of a 2D polynomial profile function of even order has been considered first. In this context, i.e. the case of a disk, this class of 
profile functions leads to designing G2D10, a set of convolution masks unbiased for even orders $N \leq 10$. The extension of this set of masks to cylinders in 3-D gives the set of masks G3D10. These masks maintain the unbiased properties of G2D10 when the cylinder lies along one of the main axes of the 3-D digital image, thus fulfilling the given objectives.

Various experiments have been carried out to assess the validity of the approach proposed. Applied to synthetic data coherent with the initial hypothesis, i.e. evenorder polynomial profile functions and the cylinder axis lining up on one of the reference mask axes, the G2D10 and G3D10 masks provide exact estimations of the direction toward the axis for $N=2 . n \leq 10$. Even in the case of odd-order polynomial profile functions, these masks still provide good estimates. They minimize the orientation bias and are at least sub-optimal.

In the more general case of noisy data, oblique cylinders or odd-order polynomial profile functions, the estimators presented in this paper will not provide exact solutions. Nevertheless, G3D10 provides accurate estimations and constantly outperforms the Prewitt, Sobel and Cross gradient masks. In the cases referred to above, other approaches might be considered in order to optimize the masks. However, obtaining unbiased orientation estimation may not be an attainable goal. These accurate results can easily be combined with the radius estimation of the object, which is directly obtained from the derivative components of the gradient. Furthermore, this combination can be used as the initial step of a segmentation algorithm. The integration of axis location estimation and cylinder segmentation in 3-D digital images is currently in progress.

\section{Acknowledgements}

The authors wish to thank "Région Aquitaine” for financial support through ARA association (“Aquitaine Rentrée Atmosphérique”), and Lee Valente for her valuable help in writing this paper.

\section{References}

[1] O. Coindreau, G.L. Vignoles and J.-M. Goyhénèche, "Multiscale X-ray CMT of C/C composites : a tool for properties assessment”, Ceram. Trans. Vol. 175, 2005, pp. 77-84.

[2] H. Bosmans, G. Wilms, S. Dymarkowski and G. Marchal, "Basic principles of MRA”, European Journal of Radiology, V. 38, V.1, 2001, pp. 2-9. 
[3] O. Coindreau and G.L. Vignoles, "Assessment of structural and transport properties in fibrous C/C composite performs as digitized by X-ray CMT. Part I: Image acquisition and geometrical properties”, Journal of Materials Research, 20, 2005, pp. 2328-2339.

[4] F. Plouraboué, P. Cloetens, C. Fonta, A. Steyer, F. Lauwers, J.-P. Marc-Vergnes, "High resolution X-ray imaging of vascular networks”, J. Microsc., Vol. 215, No 2, 2004, pp.139-148.

[5] N. R. Pal and S. K. Pal, “A review of image segmentation techniques”, Pattern recognition, Vol.6, No 9, 1993, pp. 1277-1294.

[6] S. Lakare, “3D Segmentation Techniques for Medical Volumes”, Center for Visual Computing, Department of Computer Science, State University of New York, http://www.cs.sunysb.edu/ mueller/teaching/cse616/sarangRPE.pdf, 2000.

[7] Y.L. Chang and X. Li, “Adaptive image region growing”, IEEE Trans. On Image Processing, V.3, I.6, 1994, pp. 868-872.

[8] B. Verdonck, I. Bloch and H. Maître, “Accurate segmentation of blood vessels from 3D medical images”, ICIP’96 - IEEE Int. Conf. on Image Processing, Lausanne, 1996, pp. 311-314. [9] M. Hernandez Hoyos, Segmentation anisotrope 3D pour la quantification en imagerie vasculaire par résonance magnétique, PhD Thesis, 2002.

[10] G. Langs, P. Peloschek and H. Bischof, "Determining Position and Fine Shape Detail in Radiological Anatomy”, LNCS 2781, 2003, pp. 532-539.

[11] S. Winkelbach, R. Westphal, T. Goesling, "Pose estimation of cylindrical fragments for semiautomatic bone fracture reduction”, LNCS 2781, 2003, pp. 566-573.

[12] T. Rabbani and F. van den Heuvel, "Efficient Hough transform for automatic detection of cylinders in point clouds," Proc. the 11th Annual Conference of the Advanced School for Computing and Imaging (ASCI '05), Het Heijderbos, Heijen, The Netherlands, 2005.

[13] T. McInerney and D. Terzopoulos, "Deformable Models in Medical Image Analysis: A Survey”, Medical Image Analysis, 1(2), 1996, pp. 91-108.

[14] C. Xu, D.L. Phan and J.L. Prince, "Image Segmentation using Deformable Models, Handbook of Medical Imaging, SPIE Press, 2000, Vol.2, Ch. 3.

[15] T. F. Chan and L. A. Vese, “Active contour and segmentation models using geometric PDE's for medical imaging”, Geometric Methods in Bio-Medical Image Processing, Series: Mathematics and Visualization, Springer, 2002, pp.63-75.

[16] L.D. Cohen and I. Cohen, "Finite element methods for active contour models and balloons for 2D and 3D images”, IEEE Trans. Pattern Analysis Machine Intelligence, Vol 15 (11), 1993, pp. 1131-1147.

[17] K. Krissian, G. Malandrain and N. Ayache, "Model-Based Detection of Tubular Structures in 3D Images”, Computer Vision and Image Understanding, 80, 2000, pp. 130-171.

[18] J.C. Russ, Image processing handbook - second edition, Crc press, 1995.

[19] J.F. Canny, “A computational approach of edge detection”, IEEE Trans. Pattern Analysis Machine Intelligence, 8(6), 1986, pp. 679-698.

[20] F. Le Pouliquen, J-P. Da Costa, C. Germain and P. Baylou, “A new adaptive framework for unbiased orientation estimation in textured images”, Pattern Recognition, 38, 2005, pp. 20322046. 


\section{Figure legends}

Figure a: Images showing tubular or cylindrical objects; 1) vascular networks (angiography);

2) Cross section of fiber-reinforced composite material (Synchrotron X-Ray Micro Tomography).

Figure b: Cross section of a 3-D cylinder along one of the reference mask axes; example of profile function $f(x)$ in 2-D.

Figure c: Shape of the mask $m_{i}$ in the 2-D case. Zero coefficients and the signs of non-zero coefficients are shown.

Figure d: Examples of masks $m_{i}$ in the 2-D case. These masks provide the exact orientation toward the center of the circle for profile function $f(r)=r^{N}$. The masks are centered on $O=(0,0) .1)$ G2D6 with $N=6$; 2) G2D8 with $N=8$; 3) G2D10 with $N=10$.

Figure e: Example of mask $m_{i}$ in 3-D case G3D10: 1) 3-D representation of the sections; 2) mask coefficients in the plane $\left(O_{1}, \vec{k}, \vec{j}\right)$ with $\left.O_{1}=(1,0,0) ; 3\right)$ mask coefficients in the plane $\left(O_{2}, \vec{k}, \vec{j}\right)$ with $O_{2}=(2,0,0)$. For a complete representation of $m_{x}$, consider the same section as for 2) and 3$)$ in the planes $\left(O_{-1}, \vec{k}, \vec{j}\right)$ and $\left(O_{-2}, \vec{k}, \vec{j}\right)$ with respectively $O_{-1}=(-1,0,0)$ and $O_{-2}=(-2,0,0)$, and with negative coefficients.

Figure f: Error $\Delta_{\theta}$ in the case of a cylindrical profile. This error is the angle between the orientation unit vector $\vec{v}_{n}$ and the unit normal vector to the cylinder axis $\vec{n}=\frac{\overrightarrow{M H}}{r}$ with $H$ the orthogonal projection of the current point $M$ on the cylinder axis and $r=\|\overrightarrow{M H}\|$.

Figure g: Masks used in section 4: 1) 3-D shape of the mask $m_{x} ; .2$ ) Section of Prewitt mask; 3) Section of Sobel mask; 4) Section of cross mask. The sections are in the plane $\left(O_{1}, \vec{k}, \vec{j}\right)$ with $O_{1}=(1,0,0)$. For a complete representation of the mask $m_{i}$, consider the same sections in the plane $\left(O_{-1}, \vec{k}, \vec{j}\right)$ with $O_{-1}=(-1,0,0)$ and with negative coefficients.

Figure h: Variation of the maximal angle error $\Delta_{\theta \max }$ (deg.) for various angles $\varphi$ (deg) and $\theta=90 \mathrm{deg}$. with $\varphi$ and $\theta$ defining the axis equation in spherical coordinates and for profile function of even order $N=10$. Results for G3D10, Prewitt, Sobel and cross masks are shown. Figure i: Variation of the maximal angle error $\Delta_{\theta \max }$ (deg.) estimated for pixels with the axis distance $r=3 \pm 0.5$ for various Signal to Noise Ratio. $\theta=90 \mathrm{deg}$ and $\varphi=63 \mathrm{deg}$. The signal is the gradient norm at the axis distance $r=3$. The noise is white and Gaussian. 
Figure j: Variation of the maximal angle error $\Delta_{\theta \max }$ (deg.) for G3D10, Prewitt, Sobel and Cross masks for profile function of odd order $N=9$; 1) For various angles $\varphi$ (deg.) and $\theta=90$ deg.;

2) For pixels with the axis distance $r=3 \pm 0.5$ for various Signal to Noise Ratio. $\theta=90 \mathrm{deg}$. and $\varphi=63 \mathrm{deg}$. The signal is the gradient norm. Distance to the axis is $r=3$. The noise is white and Gaussian.

Figure k: G3D10 gradient vectors projected on the corresponding fiber-reinforced composite material. The magnitude of the gradient is not shown. The 3D block results from Synchrotron XRay Micro Tomography. 
Table legends

\begin{tabular}{|l|l|l|l|l|}
\hline$\Delta_{\theta \max }(\mathrm{deg})$ & G3D10 mask & Sobel mask & Prewitt mask & Cross mask \\
\hline mean & 3.49 & 12.86 & 16.26 & 20.67 \\
\hline maximum & 5.73 & 15.74 & 19.5 & 31.01 \\
\hline minimum & $5.66 \mathrm{e}-13$ & 4.56 & 6.78 & 8.91 \\
\hline
\end{tabular}

Table a: Orientation error estimations (deg.) for G3D10, Sobel, Prewitt and Cross masks. For each mask, the table shows the mean, the minimum and the maximum errors. 


\section{Figures}

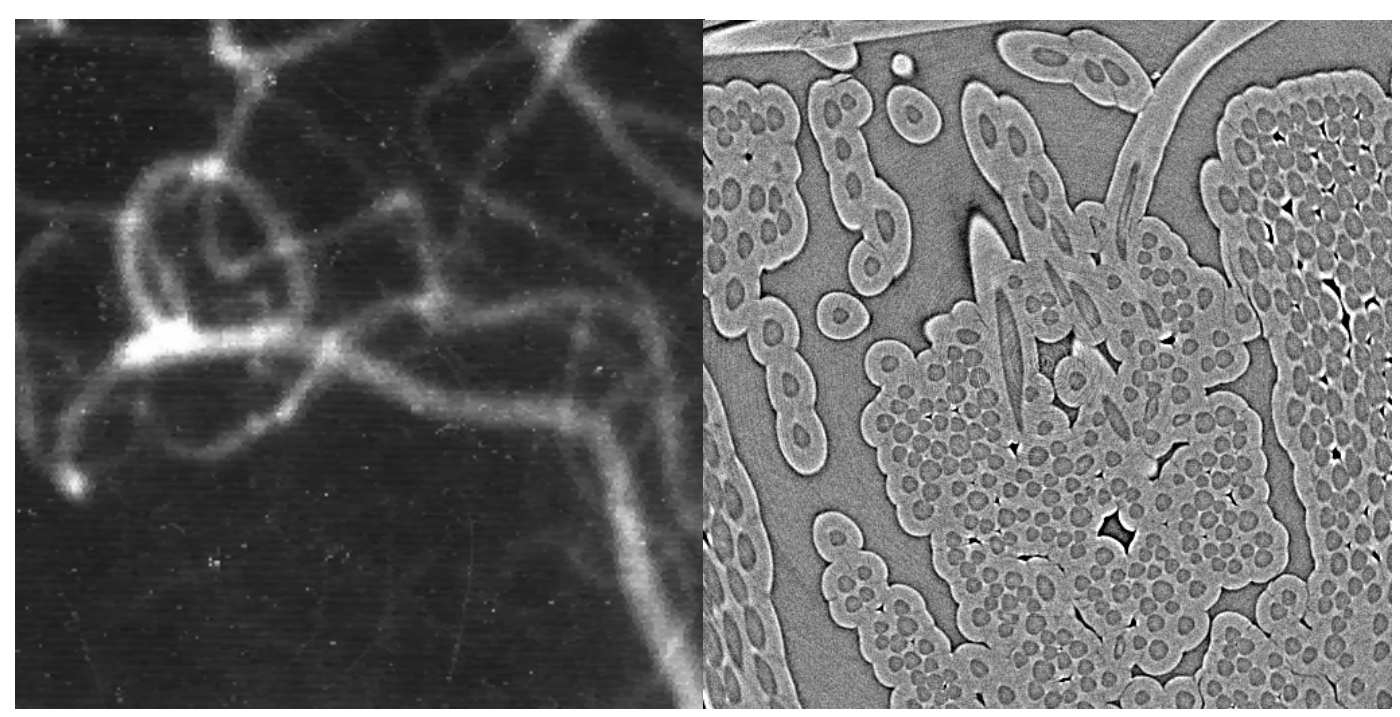

1

2

Figure a: Images showing tubular or cylindrical objects; 1) vascular networks (angiography);

2) Cross section of fiber-reinforced composite material (Synchrotron X-Ray Micro Tomography). 


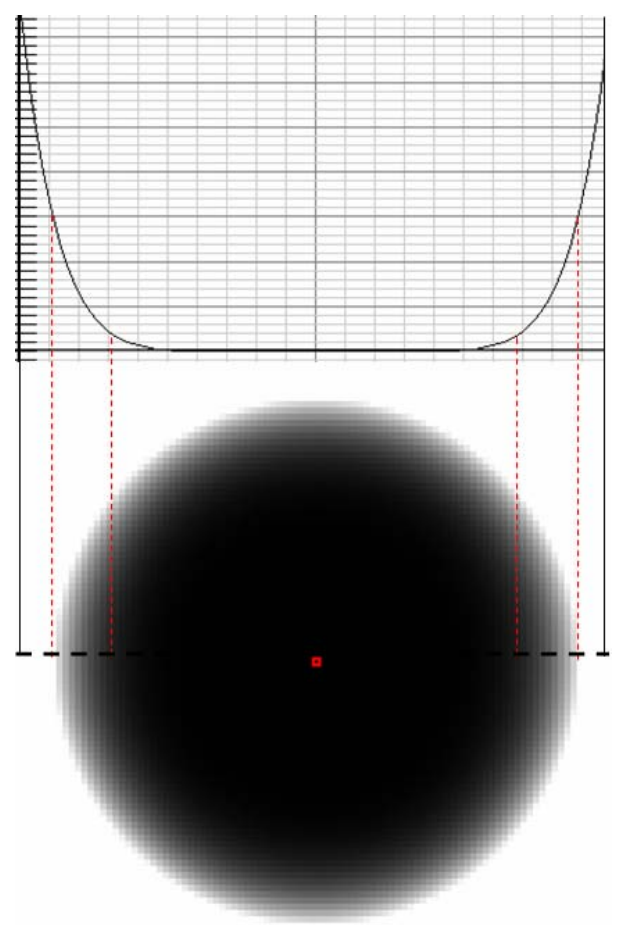

Figure b: Cross section of a 3-D cylinder along one of the reference mask axes; example of profile function $f(x)$ in 2-D. 


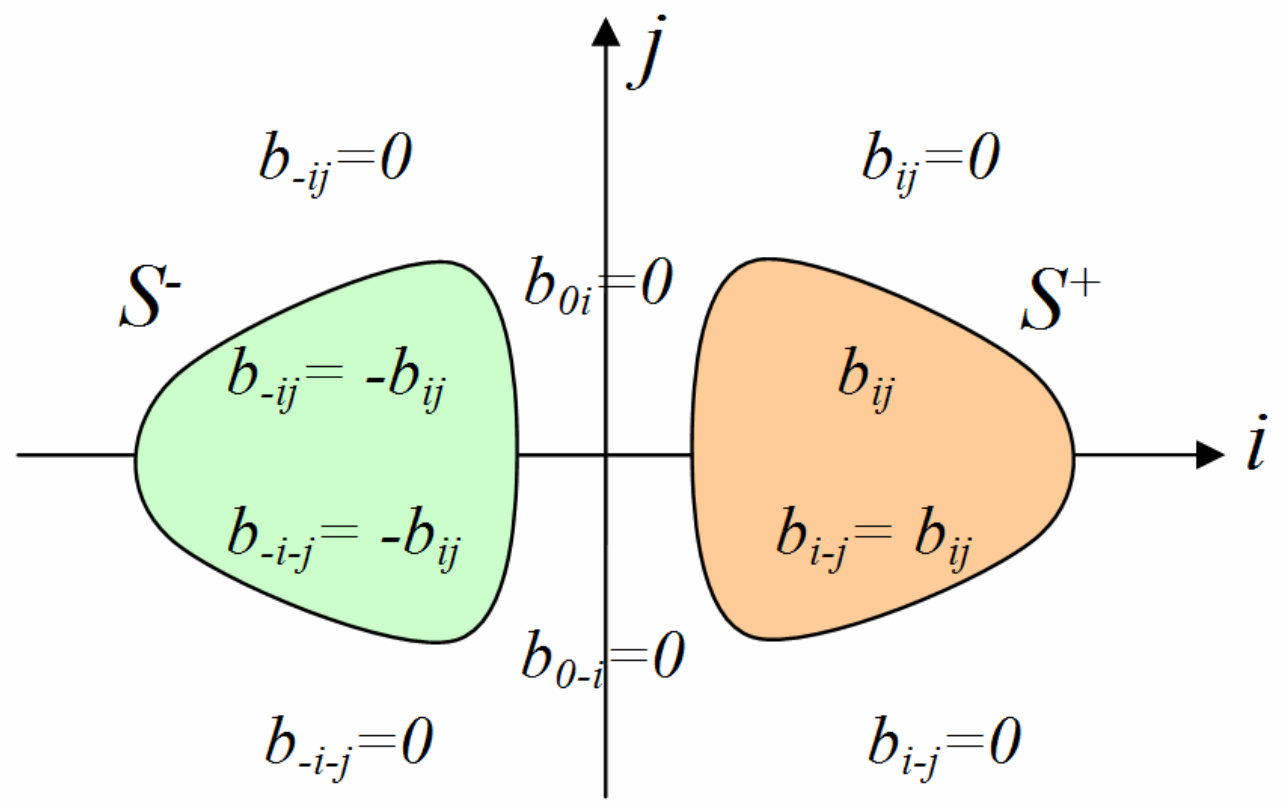

Figure c: Shape of the mask $m_{i}$ in the 2-D case. Zero coefficients and the signs of non-zero coefficients are shown. 


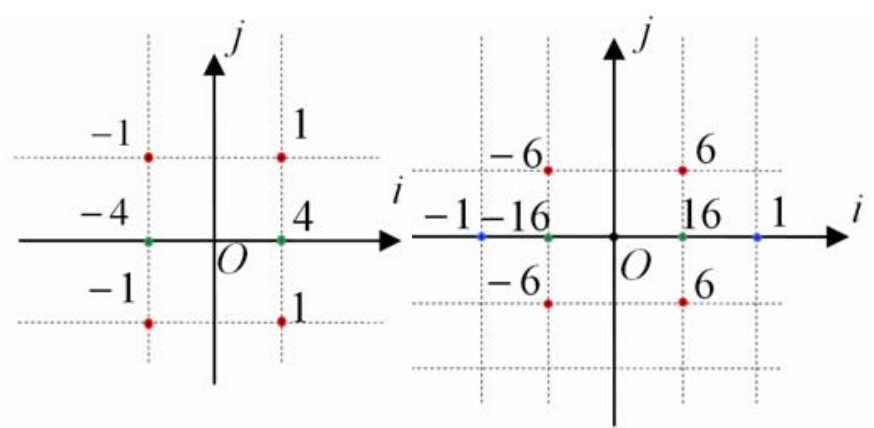

1

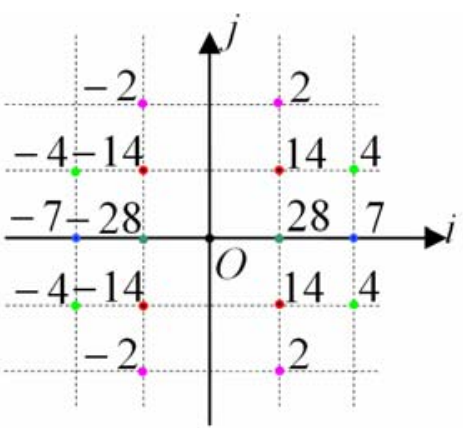

3

Figure d: Examples of masks $m_{i}$ in the 2-D case. These masks provide the exact orientation toward the center of the circle for profile function $f(r)=r^{N}$. The masks are centered on $O=(0,0) .1)$ G2D6 with $N=6$; 2) G2D8 with $N=8$; 3) G2D10 with $N=10$. 


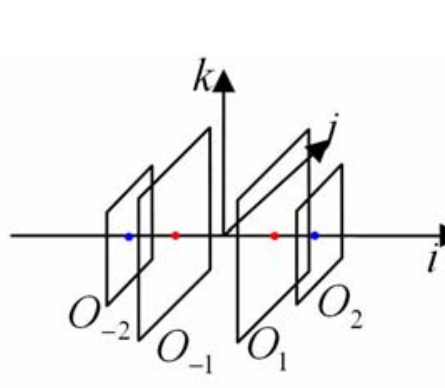

1

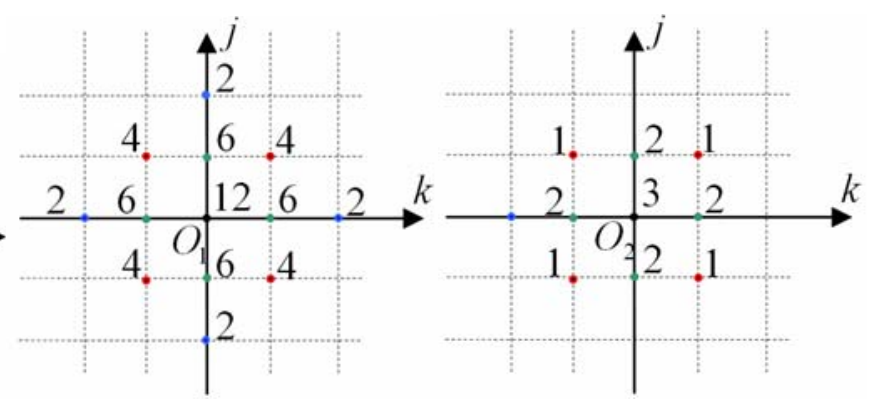

2

3

Figure e: Example of mask $m_{i}$ in 3-D case G3D10: 1) 3-D representation of the sections; 2) mask coefficients in the plane $\left(O_{1}, \vec{k}, \vec{j}\right)$ with $\left.O_{1}=(1,0,0) ; 3\right)$ mask coefficients in the plane $\left(O_{2}, \vec{k}, \vec{j}\right)$ with $O_{2}=(2,0,0)$. For a complete representation of $m_{x}$, consider the same section as for 2) and 3$)$ in the planes $\left(O_{-1}, \vec{k}, \vec{j}\right)$ and $\left(O_{-2}, \vec{k}, \vec{j}\right)$ with respectively $O_{-1}=(-1,0,0)$ and $O_{-2}=(-2,0,0)$, and with negative coefficients. 


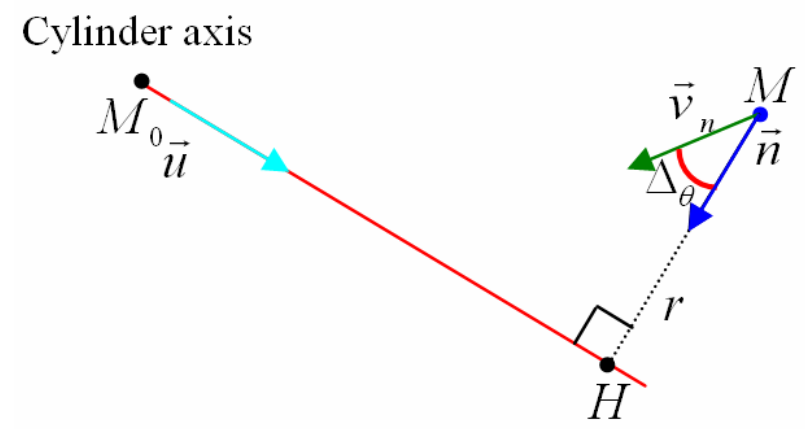

Figure f: Error $\Delta_{\theta}$ in the case of a cylindrical profile. This error is the angle between the orientation unit vector $\vec{v}_{n}$ and the unit normal vector to the cylinder axis $\vec{n}=\frac{\overrightarrow{M H}}{r}$ with $H$ the orthogonal projection of the current point $M$ on the cylinder axis and $r=\|\overrightarrow{M H}\|$. 


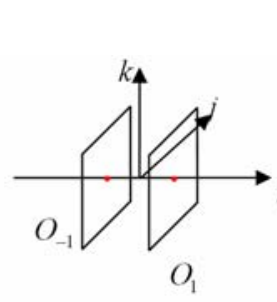

1

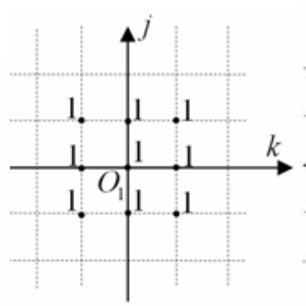

2

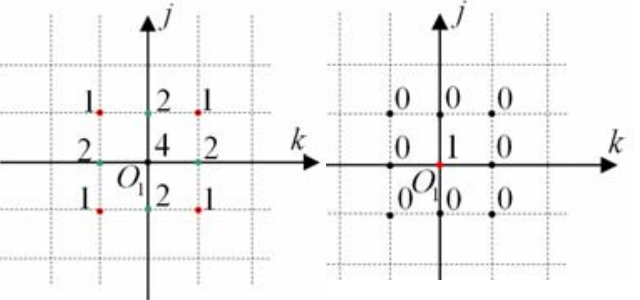

3
4

Figure g: Masks used in section 4: 1) 3-D shape of the mask $m_{x} ;$. 2) Section of Prewitt mask;

3) Section of Sobel mask; 4) Section of cross mask. The sections are in the plane $\left(O_{1}, \vec{k}, \vec{j}\right)$ with $O_{1}=(1,0,0)$. For a complete representation of the mask $m_{i}$, consider the same sections in the plane $\left(O_{-1}, \vec{k}, \vec{j}\right)$ with $O_{-1}=(-1,0,0)$ and with negative coefficients. 


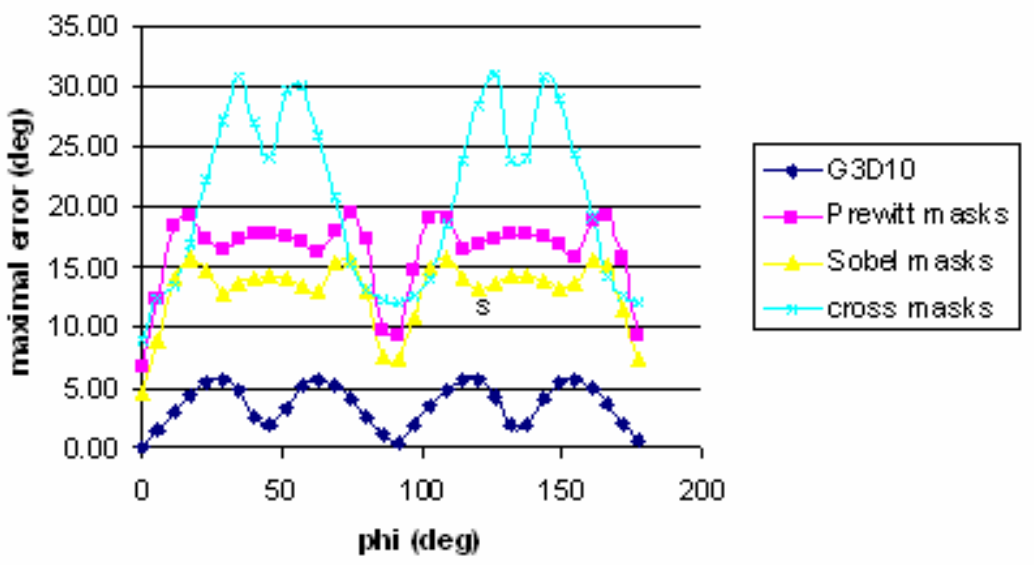

Figure h: Variation of the maximal angle error $\Delta_{\theta \max }$ (deg.) for various angles $\varphi$ (deg) and $\theta=90 \mathrm{deg}$. with $\varphi$ and $\theta$ defining the axis equation in spherical coordinates and for profile function of even order $N=10$. Results for G3D10, Prewitt, Sobel and cross masks are shown. 


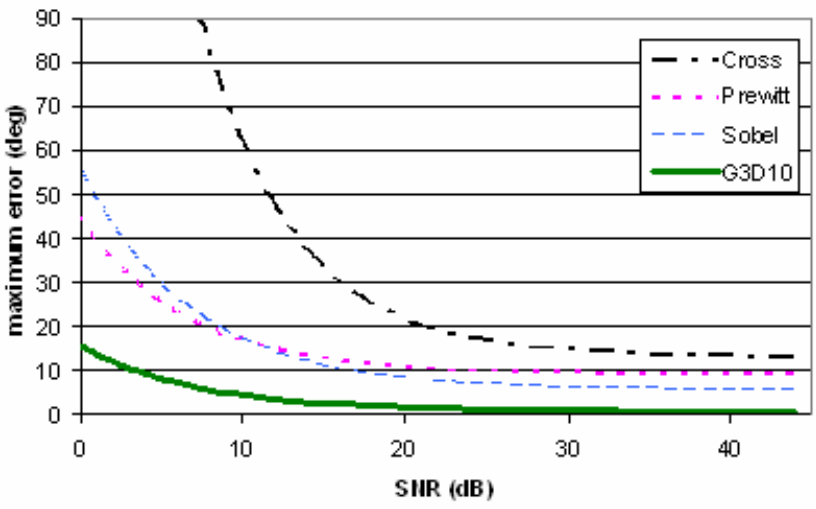

Figure i: Variation of the maximal angle error $\Delta_{\theta \max }$ (deg.) estimated for pixels with the axis distance $r=3 \pm 0.5$ for various Signal to Noise Ratio. $\theta=90 \mathrm{deg}$ and $\varphi=63 \mathrm{deg}$. The signal is the gradient norm at the axis distance $r=3$. The noise is white and Gaussian. 


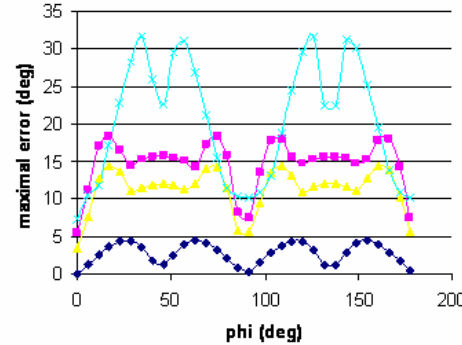

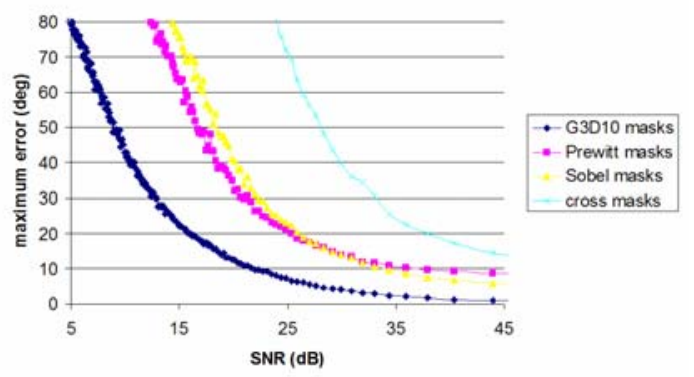

2

Figure j: Variation of the maximal angle error $\Delta_{\theta \max }$ (deg.) for G3D10, Prewitt, Sobel and Cross masks for profile function of odd order $N=9 ; 1$ ) For various angles $\varphi$ (deg.) and $\theta=90 \mathrm{deg}$.;

2) For pixels with the axis distance $r=3 \pm 0.5$ for various Signal to Noise Ratio. $\theta=90 \mathrm{deg}$. and $\varphi=63 \mathrm{deg}$. The signal is the gradient norm. Distance to the axis is $r=3$. The noise is white and Gaussian. 


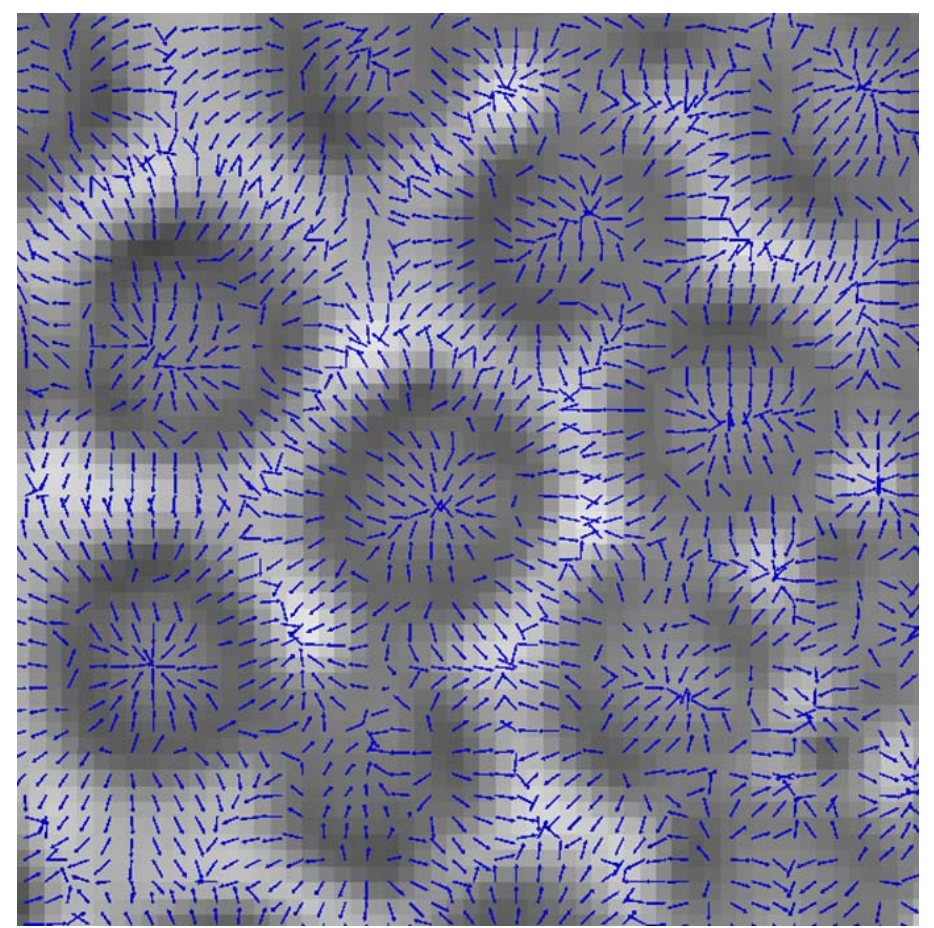

Figure k: G3D10 gradient vectors projected on the corresponding fiber-reinforced composite material. The magnitude of the gradient is not shown. The 3D block results from Synchrotron XRay Micro Tomography. 
